\title{
Spatially Localized Circular and Overlapped Feature Extraction for Gray Scale Images using Gabor Jets
}

\author{
Siddhaling Urolagin \\ Dept. of Computer Sc. and Engg., \\ Manipal Institute of Technology, \\ Manipal-576104, Karnataka India.
}

\author{
Prema K.V \\ Mody Institute of Technology and \\ Science, \\ Rajastan, India.
}

\author{
Jayakrishna $R$ \\ Dept. of Computer Sc. and Engg., \\ Manipal Institute of Technology, \\ Manipal-576104, Karnataka India.
}

\begin{abstract}
Extracting the effective features from the image for object recognition is one of the most important problems in computer vision. One of the usual methods of feature extraction is through binarization process. Binarizing the gray scale image has many disadvantages: vital brightness information loss, difficult to set proper threshold. In this paper feature-extracting method directly from the gray scale images using Gabor jets is discussed. Rather than naïve use of Gabor jets as features, a spatially localized circular and overlapped method for computing the features of Gabor jets to form feature vectors has been proposed. To demonstrate the effectiveness and efficacy of feature extraction method, classification of objects under presence of significant amount distortion using simple classifier such as K-nearest neighbor is done. A good rate of recognition is observed in the experimental results.
\end{abstract}

Keywords: Vision, Feature Extraction, Gray Scale Images, Gabor Jets.

\section{INTRODUCTION}

One of the biggest challenges of object recognition is to effectively extract features, which are able to distinguish one object from another. One of the tradition methods of feature extraction is through the binarization process. In which gray scale image is binarized and then feature from it are extracted. But binarization is the tricky process and requires domain specific threshold setting. Also by binarizing the image, vital variation in the brightness of an image is lost, which may be required to effectively form feature vectors. Feature extraction method directly from the gray scale image using Gabor filters is proposed in this paper.

The Gabor filters have widely applied to image processing and computer vision applications. In the field of computer vision 2D Gabor filter applied for edge and line detection [2][3], texture classification [4] [5], and compression [6] [7], etc. Basic reason for successful application of Gabor filter in these areas is that they possess many properties such as tunable to specific orientations; spectrally localized, which allows differentiation of linear patterns occurring over a range of spatial scales or channels; spatially localized, which allows accurate identification of linear patterns within a specific orientation; it has been shown that the complex Gabor function achieves the lower bound of the uncertainty principle; the 2D Gabor filter has the form of a complex sinusoid multiplied by a 2D Gaussian filter; extracts local information from the image, as opposed to global techniques such as the Fourier transform. For the feature extraction, thee Gabor filtering provides simultaneous inspection of image both in the spatial and frequency domains. The essence of success of Gabor filters in the field of feature extraction is that they remove most of the variability in images due to variation in lighting and contrast [8]. At the same time they are robust against small shifts and deformations. [8]. The Gabor filter representation in fact increases the dimensions of the feature space such that salient features can effectively be discriminated in the extended feature space [8]. A study has been made in [1] on the use of Gabor filtering based feature extraction for the distorted and noisy image recognition. The normalized Gabor filter response at a specific spatial location can itself be used as a local feature. In [1] the authors have proposed the use of global Gabor features, which is summation of the response over whole image.

A new method for feature extraction using Gabor filters directly on the gray scale images is proposed. A set of Gabor filters with different parameters is considered. Given a gray scale image is convoluted with set of the Gabor filters to produce Gabor jets. One might consider forming feature vectors for classification by taking Gabor jets as they are. However, such a naïve use of Gabor jets in recognition is vulnerable to deformation and variation present in the object type. So a spatially localized circular and overlapped method for accumulating the features of Gabor jets to form feature vectors is proposed. In section 2 discusses introduction about Gabor filtering, in section 3 explains the noise tolerance capability of the Gabor filters. In section 4 the proposed feature extraction method explained. Proposed method for computing features in spatially localized circular and overlapped manner is explained in section 5. In section 6 the experiment results and followed by conclusion in section 7 .

\section{GABOR FILTERING}

A two-dimensional Gabor filter $\psi(x, y)$ is a sinusoidal plane wave modulated by a Gaussian envelope. It can be defined as

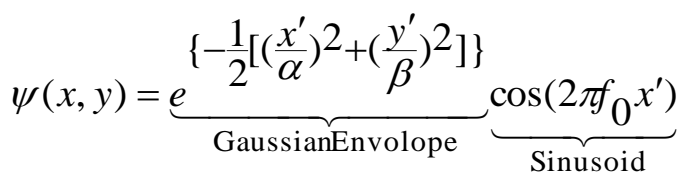

$$
\text { Where } \begin{aligned}
x^{\prime} & =x \cos \theta+y \sin \theta \text { and } \\
y^{\prime} & =y \cos \theta-x \sin \theta
\end{aligned}
$$


Where $(x, y)$ indicates $2 \mathrm{D}$ coordinates in pixels, $f_{0}$ is the frequency of the plane wave, $\theta$ is the anticlockwise rotation of the Gaussian envelope and the plane wave, $\alpha$ the sharpness of the Gaussian along the major axis parallel to the wave, and $\beta$ is the sharpness of the Gaussian minor axis perpendicular to the wave. The Gabor filters can be established in the discrete spatial domain using $\psi(x, y)$ and for given image $\xi(x, y)$ the response of the filter $r_{\xi}(x, y)$ can be calculated via convolution as

$r_{\xi}(x, y)=\psi_{f_{0}, \alpha, \beta, \theta_{i}}(x, y) * \xi(x, y)$

The Gabor filter (1) can be considered a band pass filter tuned to a specified 2D spatial frequency $f_{0}$ with a bandwidth controlled by the parameters $\alpha$ and $\beta$ and on a specified orientation $\theta$. In image processing, the Gabor filters can be used as spatially localized frequency sensitive feature extractors where the localization is realized by computing the filter response at each spatial point.

\section{NOISE TOLERANCE}

The Gabor filter can be used for low-level feature extraction. When the intended features can be constructed from the responses of the Gabor filters, the Gabor filtering provides an effective method due to its optimality in terms of minimal joint uncertainty in the spatial and frequency domains. In addition, frequency domain features have been found to be distinctly more robust than features in the presence of additive noise [9]. Still also the advantages of spatial processing can be utilized the Gabor filters.

Having the Gaussian shape in the both domains, the Gabor filters can be considered as spatially concentrated band pass filters that are localized in the frequency and spatial domains. Thus, disturbances in distinctly different location, either in the frequency or spatial domains, do not affect the filter responses. For examples, low frequency filters are tolerant for high frequency noise, such as the salt and pepper or white noise.

The Gabor filter response is sensitive to changes $\mathrm{n}$ the input image $\xi(x, y)$, but preferably a small change in the input should cause only a small change in the filter response to provide a stable behavior. The stable behavior of the steerable filters, such as the Gabor filters, can be achieved by relaxing the orthogonality property of the selected filters [10]. Since the Gabor filters can be selected to have overlap in the spatial and frequency domains, providing an overcomplete representation, a smooth behavior of the filter responses can be realized to tolerate small changes of object pose, e.g., translation, scale, and rotation. In addition, due to the spatial concentration the Gabor filters are tolerant to small changes in illumination conditions, e.g., variation in illumination gradient.

\section{FEATURE EXTRACTION USING GABOR JETS}

Unlike the usual method of extracting the feature from the image through image binarization, a scheme of feature extraction directly from the gray image using Gabor filters is proposed as shown in fig. 1. The use of the Gabor filters contributes to freeing a tricky binarization process for cluttered images, and furthermore provides localized directional edge features, which have phase-shift invariance to edge positions. A set of Gabor filters is formed with specific parameters such as fundamental frequency, sharpness of the Gaussian along major and minor axis and different rotation angles. The gray scale object image undergoes convolution with these Gabor filter to produce the filter's response called as Gabor jets. The feature vector is obtained by accumulating the Gabor jets along projection lines in circular and overlapped local regions. The scheme of extracting spatially localized features is explained in following section. Thus produced features can be used for object recognition.

\section{SPATIALLY LOCALIZED CIRCULAR AND OVERLAPPED FEATURE EXTRACTION}

In this section proposing method for computing features in spatially localized circular and overlapped manner. A set of Gabor filters $G$ each filters is formed by different parameters is employed.

$$
G=\left\{\psi_{f_{0}, \alpha, \beta, \theta_{i}}^{i}(x, y)\right\}
$$

$$
\text { Where } \theta_{i}=i \pi / 4 \text { and } 0 \leq i \leq 3 \text {. }
$$

The spatially localized feature extraction is carried out as follows

1. Normalize the given image $\xi(x, y)$ size to

$$
0 \leq x<m \text { and } 0 \leq y<n .
$$

2. Carry out the image $\xi(x, y)$ convolution with the filter $\psi_{f_{0}, \alpha, \beta, \theta_{i}}^{i}(x, y)$ to produce the filter's response $r_{\xi}^{i}(x, y)$, which is of same size as input image.

$$
r_{\xi}^{i}(x, y)=\psi_{f_{0}, \alpha, \beta, \theta_{i}}^{i}(x, y) * \xi(x, y)
$$

(6)

$$
\text { for } 0 \leq i \leq 3 \text {. }
$$




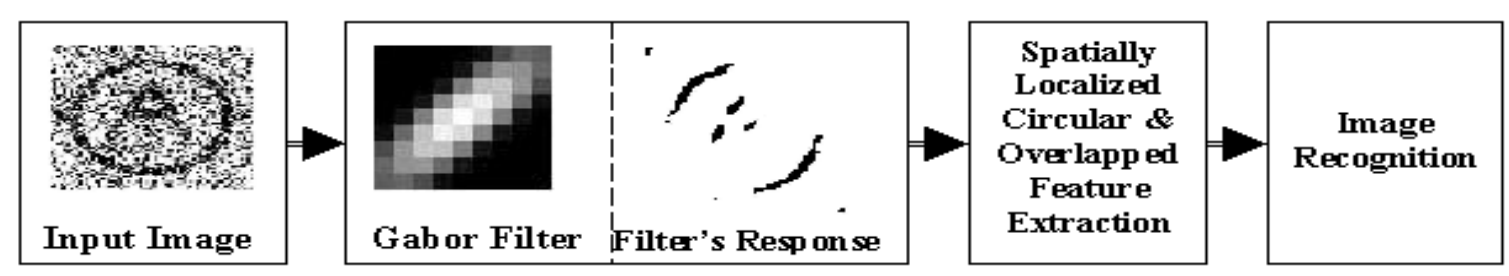

Fig1: The schematic diagram of the proposed feature extraction method using Gabor Jets.

3. Let $d$ be the division factor, which divides entire response $r_{\xi}^{i}(x, y)$ into $d \times d=d^{2}$ number of blocks.

4. A subimage $S(x, y)$ from each block is formed and adding to it $\lambda$ number of neighboring pixels from it's three neighboring blocks as shown in fig. 2(a). For blocks at the extreme end, $\lambda$ number of pixels is taken from the neighboring blocks in a circular manner as shown fig. 2(b). The size of thus formed subimage $S(x, y)$ is $m^{\prime} \times n^{\prime}$ where $m^{\prime}=m / d+\lambda$ and $n^{\prime}=n / d+\lambda$.

5. Next, accumulate $\eta$ number of directional features from each subimage $S^{(i, b)}(x, y)$ based on the $\theta_{i} \quad$ where $\quad \theta_{i}=i \pi / 4, \quad 0 \leq i \leq 3 \quad$ and $0 \leq b<d^{2}$.

a. When $i=0, \theta=0$, features are extracted along the horizontal direction

$v_{k+b \eta+i d^{2} \eta}=\sum_{x=k \delta}^{x<(k+1) \delta} \sum_{y=0}^{y<n^{\prime}} S^{(i, b)}(x, y)$

b. When $i=1, \theta=\pi / 4$, feature are extracted along $v_{k+b \eta+i d^{2} \eta}=\sum_{x=0}^{\substack{\text { principle } \\ x<m^{\prime}}} \sum_{y={ }_{0}\left[n^{\prime}-2(k+1) \delta+x+1\right]^{n^{\prime}}}^{\left.y<n^{\prime}-2 k \delta+x\right]^{n^{\prime}}} S^{(i, b)}(x, y)$

(9) c. When i=2, $\theta=\pi / 2$, features are extracted along the vertical direction

$$
v_{k+b \eta+i d^{2} \eta}=\sum_{x=0}^{x<m^{\prime}} \sum_{y=k \delta}^{y<(k+1) \delta} S^{(i, b)}(x, y)
$$

(10)

d. When $\mathrm{i}=3, \theta=3 \pi / 4$, feature are extracted along the secondary diagonal

$$
v_{k+b \eta+i d^{2} \eta}=\sum_{x=0}^{x<m^{\prime} y<_{0}[2(k+1) \delta-x-1]^{n^{\prime}} \sum_{y=0} S^{(i, b)}(x k-x]^{n^{\prime}}}(x, y)
$$

(11)

Repeat above steps for all $0 \leq k<\eta$, $0 \leq b<d^{2}$, where $0 \leq i \leq 3$

Here introducing range defining operator ${ }_{l}[\varpi=f(\zeta)]^{u}$ on function $\varpi=f(\zeta)$ such that,

$$
a=\left\{\begin{array}{c}
u \text { if } f(\zeta)>u \\
l \text { if } f(\zeta)<l \\
f(\zeta) \text { otherwise }
\end{array}\right.
$$

7. At the end of the feature extraction for each response $r_{\xi}^{i}$ where $0 \leq i \leq 3$ a vector $V$ of size $|V|=|G| \times d^{2} \times \eta$ will result.

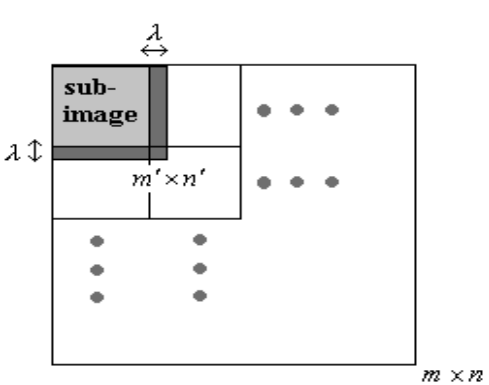

Fig 2(a): Sub-image formation.

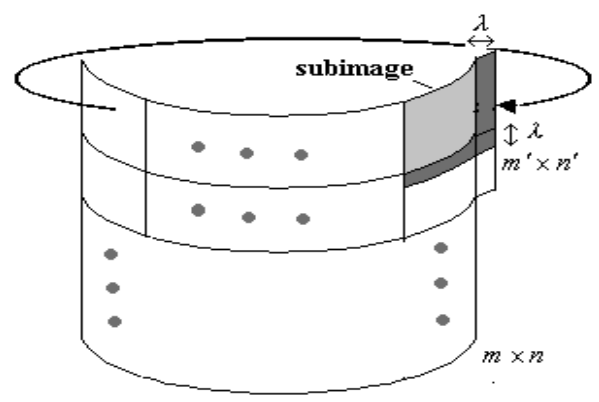

Fig 2(b): Sub-image formation at extreme image. 


\section{EXPERIMENT AND RESULTS}

Electric symbols of [1] for object recognition are used in the experiment. These symbols can be downloaded from the address given in the [1]. There are 25 electric symbol images as shown in fig. 3 .

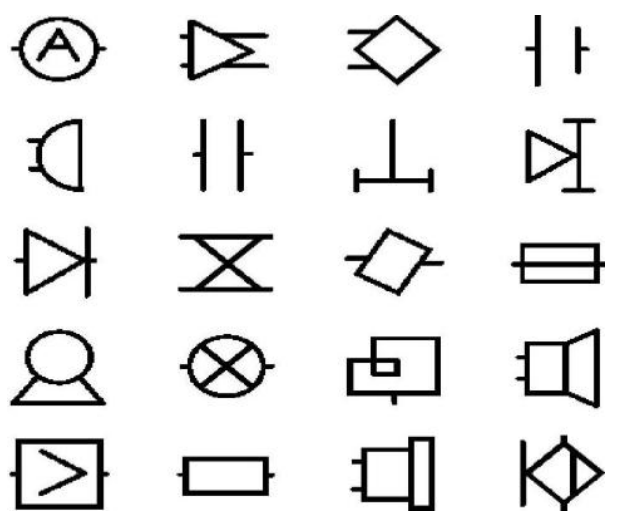

Fig3: Electric symbol images

The gray scale images are normalized to 64 X64 size. A set of Gabor filters is formed with $\alpha=4, \beta=4$, fundamental frequency is $f_{0}=0.11$ and different angles $\theta=0,45,90$, and 135 . The normalized images undergo convolution with each of the four filters to produce responses. For each responses thus produced is divided into 16 blocks. Taking one block at a time, a subimage is formed of size $20 \times 20$ by appending 4 neighboring pixels from its neighboring block as explained in section 5. From each of the subimage 4 features are accumulated along horizontal, principle diagonal, vertical or secondary diagonal direction based on the angles 0,45,90,135 respectively used for constructing the Gabor filter. Thus from the each image 256 features are accumulated.

In order to know the efficacy of the feature extraction method discussed so far, K-nearest neighbor method for classification of the images is used. K-nearest neighbor is chosen because it is simple and distance based classification method, so that emphasis on and measure performance of feature extraction technique. A base images set is formed by undistorted electric symbol images of fig. 3 along with symbol images having Gaussian noise of mean zero and variance of $0.01,0.05,0.09,0.1,0.5,0.9,1.1,1.5,1.9,2.1$, $2.5,2.9,3.1$, and 3.5. A test images set is formed by electric symbol images having Gaussian noise of mean zero and variance ranging from 0.02 to 3.6 other than the images of base images set. The recognition of test images is carried out using K-nearest neighbor based on base images set. The Knearest neighbor finds the $\mathrm{K}$ samples of base set having minimum distance between the features of test image to the features of base set images. The given test image assigned the class label of the base set image having the majority among the K samples.

The fig. 4 shows the recognition rate when K-nearest neighbor is used with $\mathrm{K}=3,5$ and 7 to classify test images based on the base images set. The recognition rate for test images against variance varying from 0.02 to 3.6 is shown in the fig 4. Recognition rate for all most all the cases is $100 \%$ and a minimum of $92 \%$ recognition rate is observed when $K=3$ at variance 3.6. For higher value of $K(K=5, K=7)$ better recognition rate is obtained than $\mathrm{K}=3$.

The fig. 5 shows the recognition rate for each class of the test images set. There are 25 different classes in the problem space. The fig.5 shows the recognition rate at various $\mathrm{K}$ values. Except for ammeter and battery, for all the classes recognition of $100 \%$ is observed. For ammeter class recognition rate is $86.95 \%$ when $\mathrm{K}=3,5,7$ and for battery class it is $95.65 \%$ when $\mathrm{K}=5,7$ is obtained.

\section{CONCLUSION}

In this paper new method for feature extraction directly on gray scale images is proposed. Using a set of Gabor filter and convoluted the given image to produce Gabor jets. Rather than using Gabor jets as features, which are vulnerable to deformation and variations, proposed spatially localized circular and overlap method for computing features from Gabor jets. In order to measure performance of and emphasis on proposed feature extraction method, K-nearest neighbor for classification is used, which is simple and distance based method. The recognition rate for the test images having Gaussian noise is observed to be $100 \%$ in all most all the cases. A better recognition rate is observed for all the classes of the problem space. The proposed feature extraction method for gray scale images provides better recognition results under significant amount of distortion.

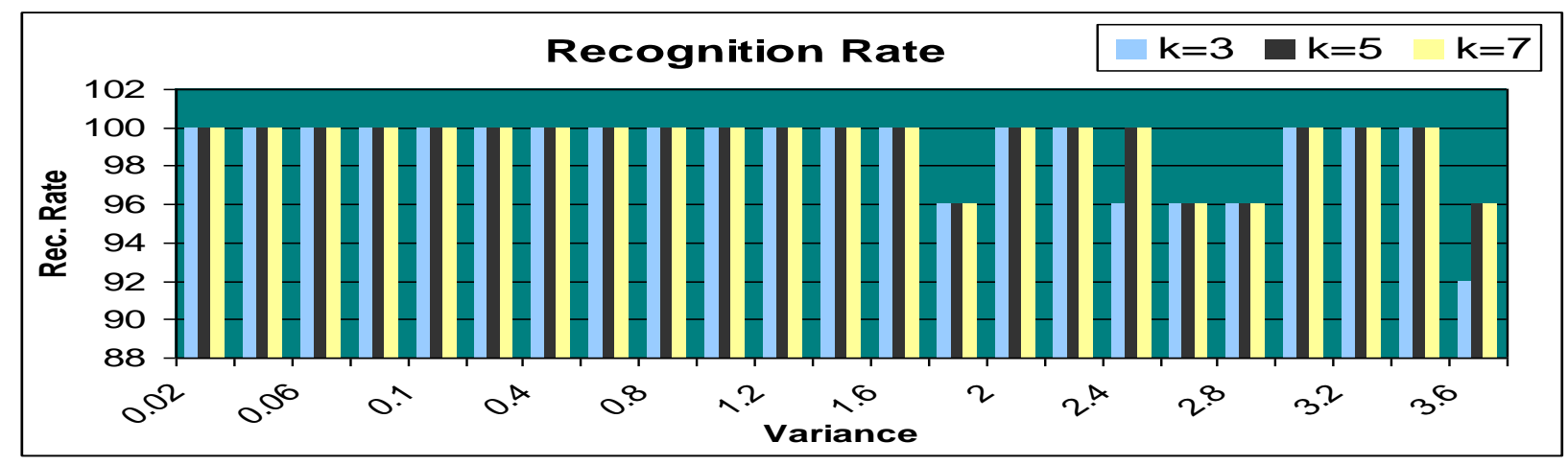

Fig 4: Recognition rate for the test images having Gaussian noise with variance from 0.02 to 3.6 


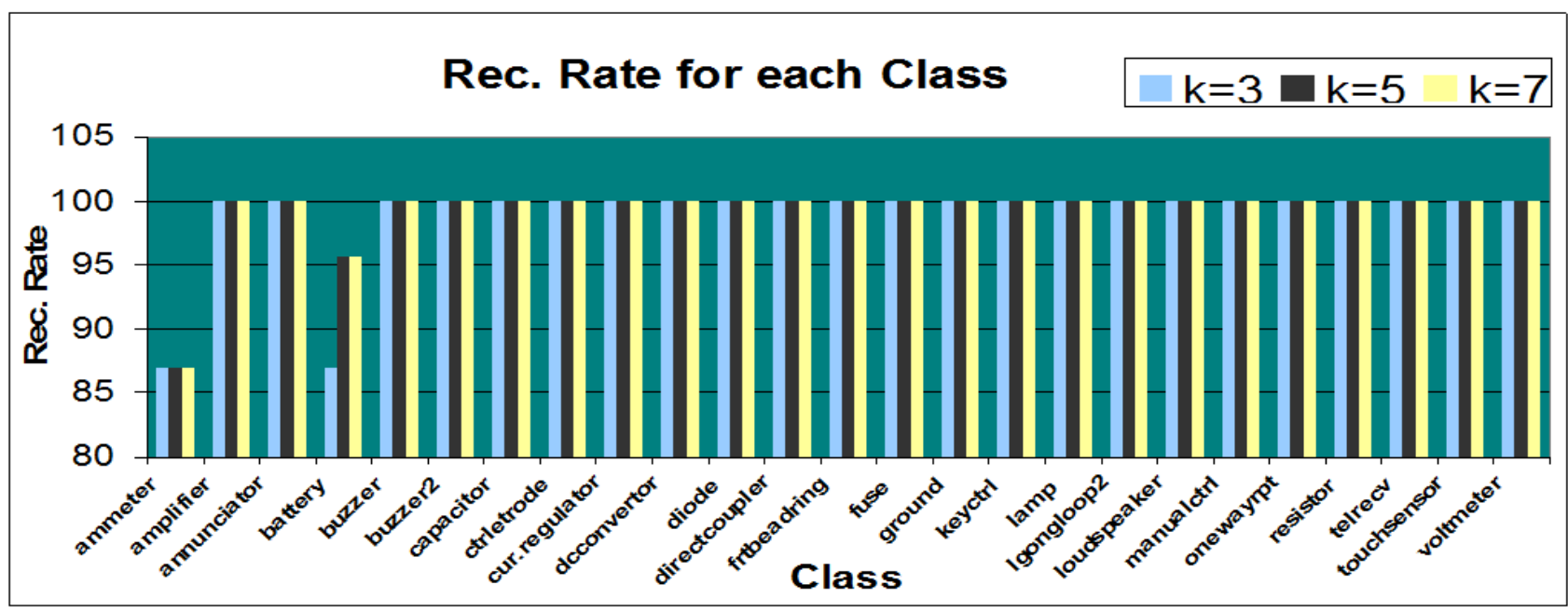

Fig 5: Recognition rate for each class of test images set

\section{REFERENCE}

[1] J. K. Kamarainen, V.Kyrki, and H. Kalviainen, Noise tolerant object recognition using gabor filtering. In 14th International Conference on Digital Signal Processing, DSP 2002, vol.2, pp. 1349-1352, 1-3 July 2002.

[2] R. Mehrotra, K. Namuduri, and N. Ranganathan, Gabor filter-bade edge detection. In Pattern Recognition, vol.25, no. 12, pp. 1479-1494, 1992.

[3] J.Chen, Y.Sato, and S.Tamura, Orientation space filtering for multiple orientation line segmentation. In IEEE Transactions of Pattern Analysis and Machine Intelligence, vol.22, pp.417-429, May 2000.

[4] A.C.Bovik, M. Clark and W.S. Geisler, Multi-channel texture analysis using localized spatial filters. In IEEE Transactions of Pattern Analysis and Machine Intelligence, vol.12, pp. 55-73, January 1990.

[5] A. K. Jain and E. Farrokhnia, Unsupervised texture segmentation using Gabor filters. In Pattern Recognition, vol. 24, no. 12, pp. 1167-1186, 1991.
[6] M. Porat and Y.Y. Zeevi, The generalized Gabor scheme of image representation if biological and machine vision. In IEEE Transactions of Pattern Analysis and Machine Intelligence, vol. 10, pp. 452-468, July 1988.

[7] T.S. Lee, Image representation using 2D Gabor wavelets. In IEEE Transactions of Pattern Analysis and Machine Intelligence, vol. 18, pp. 959-971, October 1996.

[8] Lim. T.R., Guntoro, A.T.; Car recognition using Gabor filter feature extraction. In Asia-Pacific Conference on Circuits and Systems APCCAS '02, vol. 2, pp.451-455, 28-31 Oct. 2002

[9] S.S. Liu and M. Jernigan, Texture analysis and discrimination in additive noise. In computer Vision, Graphics, and Image Processing, vol. 49, pp. 52-67, 1990.

[10] E.P. Simoncelli, W.T. Freeman, E.H.Adelson, and D.J.Heeger, Shiftable multiscale transforms. In IEEE Transactions on Information Theory, vol. 38,pp. 587-607, Mar. 1992. 